\title{
Office diagnostic smart hysterofiberscopy, hysmartscopy, using mobile technology: A single center experience and analysis of diagnostic accuracy
}

\author{
KANA IWAI ${ }^{1}$, HIROSHI SHIGETOMI ${ }^{1,2}$, KIYOSHI OKA ${ }^{1,3}$ and HIROSHI KOBAYASHI ${ }^{1}$ \\ ${ }^{1}$ Department of Obstetrics and Gynecology, Nara Medical University, Kashihara-shi, Nara 634-8522; \\ ${ }^{2}$ Aska Ladies Clinic, Nara-shi, Nara 631-0053; ${ }^{3}$ National Institutes for Quantum and Radiological Science \\ and Technology, Kitsukawa-shi, Kyoto 619-0215, Japan
}

Received October 3, 2019; Accepted November 5, 2019

DOI: $10.3892 /$ wasj.2019.26

\begin{abstract}
Office hysteroscopy is a minimally invasive procedure and provides a direct view of the endometrial cavity. This study presents the first report of a novel smartphone-based ultrathin flexible hysterofiberscopy system (currently known as office diagnostic smart hysterofiberscopy, or Hysmartscopy), for the diagnosis of intrauterine abnormalities. The Hysmartscopy system captures images using a flexible fiberscope $(0.8 \mathrm{~mm}$ in diameter) coupled with an Apple iPhone $6 \mathrm{~S}^{\mathrm{TM}}$. This study was conducted at the Department of Gynecology of Nara Medical University Hospital from February, 2015 to February, 2019. In total, 22 patients requiring a diagnostic Hysmartscopy for the investigation of intrauterine pathology were included in this study. In the first cohort, Hysmartscopy was performed in the operating room with anesthesia in 17 patients to assess the overall physician's experience during image acquisition, subjective image quality and the ease of use on a 5-point Likert scale. In the second cohort, 5 subjects further underwent Hysmartscopy without anesthesia to evaluate the feasibility and safety of this system. Images were assessed by two expert gynecological endoscopists blinded to the pathological reports. The Hysmartscopy system revealed good resolutions in the ex vivo image and color resolution. From the beginning of the procedure, the time required to capture a video was $<1 \mathrm{~min}$. The ergonomic advantage of this technique enabled the examiners to use direct visualization for image-guided diagnosis in a hand-held manner. On average, the quality of the images (4.0/5.0 on a 5-point Likert scale) may be largely sufficient to detect emergent findings. The diagnostic accuracy of Hysmartscopy was found to be 100\% (2/2) for the normal
\end{abstract}

Correspondence to: Professor Hiroshi Kobayashi, Department of Obstetrics and Gynecology, Nara Medical University, 840 Shijo-cho, Kashihara-shi, Nara 634-8522, Japan

E-mail: hirokoba@naramed-u.ac.jp

Key words: hysteroscopy, hysterofiberscopy, outpatient clinic, smartphone, technology endometrium, $71.4 \%(5 / 7)$ for endometrial polyps, $62.5 \%$ (5/8) for submucosal fibroids and $100 \%(5 / 5)$ for endometrial cancer. The diagnostic accuracy of Hysmartscopy was $77.3 \%$. None of the cases had any complications during and after the diagnostic procedures. Thus, the results indicate that Hysmartscopy is a patient-friendly technique without the need for anesthesia and cervical dilation in an office setting. To the best of our knowledge, this study is first proof of concept pilot study of Hysmartscopy. Hysmartscopy may prove to be a simple, convenient, non-invasive, accurate and well-tolerated procedure for the diagnosis of intrauterine abnormalities that can be performed within an outpatient clinic.

\section{Introduction}

Transvaginal sonography, hysteroscopy and magnetic resonance (MR) imaging are diagnostic tools for intrauterine abnormalities (1). Hysteroscopy is a mainstay diagnostic method for women with an abnormal uterine cavity and bleeding $(2,3)$. Traditionally, hysteroscopy often requires the use of mechanical or pharmaceutical dilatations of the cervix before this procedure under local or general anesthesia in the operating room (4). Although this is considered a safe procedure, patients are at risk of developing potential complications, such as bleeding, perforation, infection, fluid overload, incomplete resection and intrauterine adhesions (2). Therefore, very few gynecologists perform hysteroscopy in an outpatient setting. Recent advances in technology and improved techniques have turned hysteroscopy into a common outpatient procedure which can be performed in an office setting (outpatient hysteroscopy or office hysteroscopy) (3). Outpatient hysteroscopy is a minimally invasive procedure and offers a direct visualization of the endometrial cavity (5). No anesthetic or local anesthesia allows the use of outpatient settings, without taking the patient to the operating room (6). The number of outpatient hysteroscopy procedures is increasing (6).

The authors have previously developed an ultrathin fiberscopic imaging system for laser surgery that may be an important instrument for clinical use during office-based hysteroscopy (7). This device consists of a custom $1.1 \mathrm{~mm}$ in diameter flexible fiberscope and ytterbium laser-supported 
ablation system (composite-type optical fiberscope) to achieve accurate laser irradiation for minimally invasive procedures of intrauterine disease (7). In the present study, the authors further developed an optical ultrathin hysterofiberscopic imaging system with a flexible fiberscope $(0.8 \mathrm{~mm}$ in diameter $)$ for the diagnosis of intrauterine abnormalities. The novelty of this method concerns the development of the slimmest fiber hysteroscope. In consequence, its use does not require cervical dilatation and anesthesia which causes discomfort, and is a convenient and patient-friendly technique. In addition, recent advances and the widespread availability of smartphones render the utility of this technology practical and promising in medicine. The smartphone alone allows for image acquisition, image capture, video capture, image storage and projection. A smartphone replaces the camera and video receiver box, and no separate video receiver box is required. This study provides an initial report of a novel docking system that optimizes the coupling of a smartphone, the Apple iPhone $6 \mathrm{~S}^{\mathrm{TM}}$, with an office diagnostic, small-diameter hysterofiberscopy, 'Hysmartscopy' (a smart hysterofiberscopy).

The aim of this study was to evaluate the image quality for the diagnosis of intrauterine abnormalities and to assess the feasibility and safety of the Hysmartscopy system.

\section{Materials and methods}

System setup and design. The authors of this study developed an optical flexible fiberscope system for the diagnosis of intrauterine abnormalities. The smartphone-based intrauterine imaging system was a flexible fiberscope $(0.8 \mathrm{~mm}$ in diameter) coupled with an Apple iPhone 6 $\mathrm{S}^{\mathrm{TM}}$ (Apple Inc.) in camera mode. The Hysmartscopy system functions on the principles of a direct fiberscope and exploits the autofocus capability of the iPhone camera. For maximal image quality, this system provides a centered image on the iPhone camera with an $8 \mathrm{X}$ optical zoom. It is an ultrathin and light weight, simple system that turns a smartphone into a portable camera capable of capturing and uploading high-quality images and videos of the intrauterine pathologies via the Hysmartscopy system. An overview of the whole system is illustrated in Fig. 1. The information of the Hysmartscope is as follows: Optical system [field of view $\left(90^{\circ}\right)$, direction of view $\left(0^{\circ}\right.$ forward viewing)], insertion portion [outer diameter $(0.8 \mathrm{~mm})$, total length $(210 \mathrm{~mm})]$, and instrument channel [minimum visible distance $(3 \mathrm{~mm})$ ]. When Hysmartscopy with a $90^{\circ}$ field of view was used at a focal distance of $5 \mathrm{~mm}$, there was no marked difference in resolution between Hysmartscopy and conventional hysteroscopy. Fluid (saline) is instilled transcervically into the uterine cavity to provide clear visualization of uterine intracavitary pathologies. The examiners can transmit the images to a remote endoscopic specialist through a web application.

Image and color resolution. The image resolution (line pairs/mm) of the Hysmartscopy-coupled mobile device was evaluated by imaging a 1951 United States Air Force Contrast Resolution Chart Target (Edmund Industrial Optics ${ }^{\mathrm{TM}}$ ). The Hysmartscope was held 5-10 $\mathrm{mm}$ above the resolution target, and images were recorded. The color resolution/quality was evaluated using the Munsell ColorChecker Chart. The colors included the following: Red, orange, yellow, green, blue, light purple and purple. The images were reviewed and evaluated by expert endoscopists, residents and medical students. A total of 34 evaluators (13 expert endoscopists, 10 residents and 11 medical students) were asked to rate the resolution, brightness, color and overall image quality from 1 (very poor, inadequate for any diagnostic purpose) to 5 (very good, ideal quality) on a 5-point Likert scale.

Proof of concept pilot study. The study protocol was in agreement with the Helsinki Declaration for Ethical Medical Research (no. 874). Research was performed after obtaining approval from the Nara Medical University Institutional Review Board (IRB). A written informed consent was signed by each participant following a detailed explanation of the objectives and protocol of the study. This pilot study was conducted at the Department of Gynecology of Nara Medical University Hospital from February, 2015 to February, 2019. Hysmartscopy was performed based on the following 4 indications: i) An abnormal uterine cavity, as suggested by transvaginal ultrasonography and/or MR imaging; ii) subjects who underwent pathological diagnosis; iii) prior to surgery; and iv) not excluding cases with a diagnosis of a uterine malignancy. The exclusion criteria were as follows: Virginity, cervical stenosis, cervicovaginal infections, pelvic inflammatory disease and pregnancy. Demographic records for each individual, including age at diagnosis, parity, menopausal state, body mass index (BMI), past medical history, histological diagnosis, grade and stage were all retrospectively analyzed from the same database.

Diagnostic Hysmartscopy. Prior to Hysmartscopy, the participants underwent transvaginal sonography and/or MR imaging. The patient was examined in the lithotomy position. A baseline transvaginal ultrasound examination of the pelvis was performed using GE Healthcare Voluson S6 (General Electric Co.). Hysmartscopy was performed in the early to middle follicular phase of each menstrual cycle; in women who experienced irregular cycles or menopause, it was performed at any time point. The presence of free fluid in the cul-de-sac was recorded prior to Hysmartscopy to calculate saline retrograde regurgitation. At first, a flexible fiberscope $(0.8 \mathrm{~mm}$ in diameter) was assembled to relay the image onto the Apple iPhone $6 \mathrm{~S}^{\mathrm{TM}}$ in camera mode. Following preparation and draping, a sterile speculum was inserted into the vagina, the cervix was visualized and cleaned with an iodine solution, and the anterior lip was grasped with a single-toothed tenaculum if required. The subjects underwent Hysmartscopy without cervical dilatation. A catheter sheath with an outer diameter of $2.2 \mathrm{~mm}$ was inserted transcervically into the uterine cavity. The flexible fiberscope was inserted through a catheter sheath and moved through the sheath to the uterine cavity. Using a $10-\mathrm{ml}$ syringe attached to the catheter, sterile saline solution of 20 to $50 \mathrm{ml}$ was slowly instilled, and the endometrial cavity was then observed. The examiner was instructed to hold the catheter sheath with one hand (with the thumb and second and third digits) and worked from a comfortable position by aiming through the screen of the iPhone. 


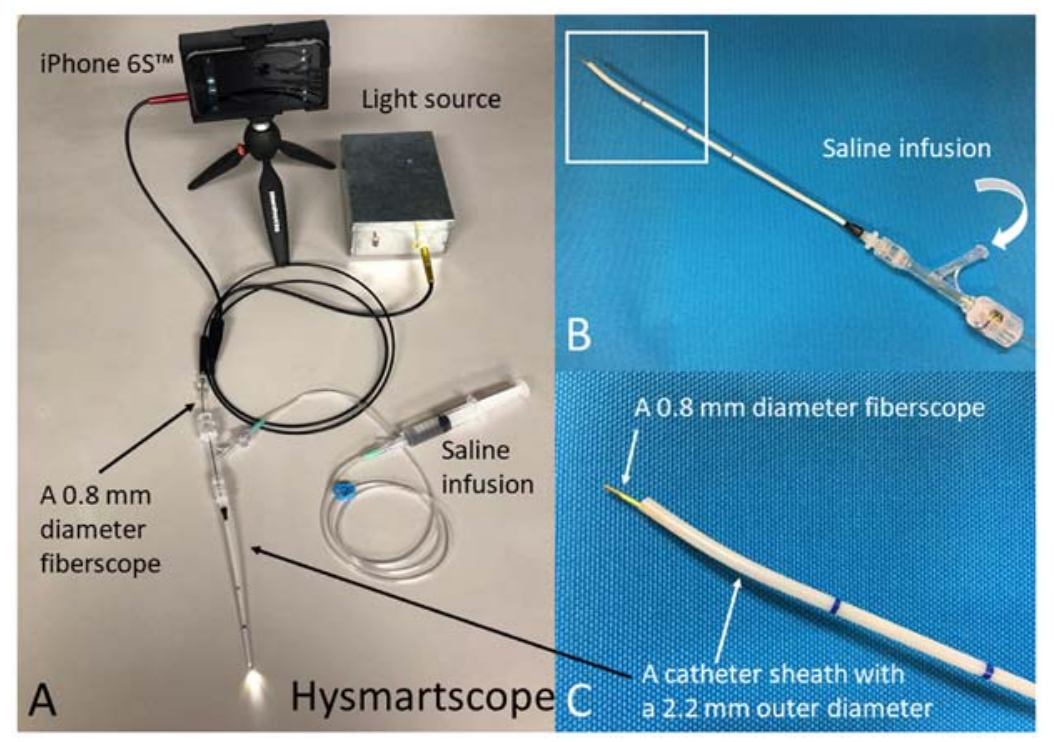

Figure 1. Setup of a typical Hysmartscopy system. (A) setup. Mechanical layout of the fiberscope and smartphone-based monitor display. The Hysmartscopy system connecting to the flexible fiberscope, $0.8 \mathrm{in} \mathrm{mm}$ diameter, with the iPhone $6 \mathrm{~S}^{\mathrm{TM}}$. (B) A catheter sheath with a $2.2 \mathrm{~mm}$ outer diameter. A $0.8 \mathrm{~mm}$ diameter fiberscope is inserted through a catheter sheath. Sterile saline is instilled into the endometrial cavity through a catheter sheath. (C) The white square lesion indicated by the white square in (B) was set to $\mathrm{x} 4$ magnification.

Image evaluation. Patients were imaged by 1 of 2 expert gynecological endoscopists (K.I. and H.S.). The uterine cavity was systematically examined and any observations were stored in electronic medical records. Acquired images and videos can subsequently be saved in the local memory and stored via secure server. The diagnostically important frames were extracted from iPhone videos recorded in full. The images collected from each patient were reviewed and evaluated by 2 endoscopists. The evaluators were blinded to the histological results.

In total, 22 patients requiring a diagnostic Hysmartscopy for the investigation of intrauterine pathology were included in this study. Following the Hysmartscopic procedures, all the patients received surgery, including total hysterectomy $(n=7)$ or transcervical resection $(n=15)$. As a preliminary evaluation from February, 2015 to December, 2017 (the first cohort), Hysmartscopy was performed in a conventional operating room under general anesthesia on 17 patients (Propofol, $n=15$; and Sevoflurane, $n=2$ ). The pathological results revealed endometrial benign polyp $(n=4)$, submucosal fibroids $(n=6)$, endometrial cancer $(n=5)$ and normal endometrium $(n=2)$. In the second cohort from January, 2018 to February, 2019, Hysmartscopy was further validated in 5 additional subjects with endometrial benign polyp $(\mathrm{n}=3)$ and submucosal fibroids $(n=2)$ without anesthesia and cervical dilatation. Finally, a total of 22 cases were analyzed in this study and these were divided into 4 groups. These included the endometrial benign polyp $(n=7)$, submucosal fibroid $(n=8)$, endometrial cancer $(n=5)$ and normal endometrium $(\mathrm{n}=2)$ groups. The patients underwent an outpatient evaluation. The results of Hysmartscopy and the pathological reports of endometrial biopsy were compared, and the diagnostic accuracy of Hysmartscopy for normal endometrium, endometrial polyps, submucosal fibroids and endometrial cancer was evaluated. The evaluators were asked to rate the resolution, brightness, color and overall image quality on a 5-point Likert scale.
Statistical analysis. Statistical analyses were performed using SPSS Statistics version 22 (International Business Machines Corp.). Data distribution was verified by the Shapiro-Wilk test, presenting that all cases exhibited normal distribution (data not shown). Age and BMI among groups were analyzed by one-way ANOVA, followed by Tukey's honestly significant difference test as a post-hoc test. To assess nulliparous and premenopausal state among 4 groups, Fisher's exact test was used. A P-value $<0.05$ was considered to indicate a statistically significant difference.

\section{Results}

Ex vivo imaging. Images of the Hysmartscopy system were recorded for the iPhone camera by imaging the color target and the resolution target. On average, the quality of the images taken by 13 expert endoscopists (median, 4.0/5.0 on a 5-point Likert scale; mean, 3.4/5.0), 10 residents (median, 4.0/5.0; mean, 3.7/5.0) and 11 medical students (median, 3.0/5.0; mean, 3.5/5.0) was sufficient to detect emergent findings, while it is somewhat difficult to detect the subtle findings (data not shown). For 2 expert endoscopists (K.I. and H.S.), the photographic quality on a 5-point scale was ranked 4, 'somewhat agree' (data not shown). Thus, the endoscopic image quality overall was within the diagnostically acceptable range.

In vivo imaging. The major clinical and pathological characteristics are listed in Table I. The age of the studied patients was $42.9 \pm 9.7$ years (mean \pm standard deviation; range, 28 to 67 years). Age was higher in subjects with endometrial cancer when compared to the other subjects, which was statistically significantly different $(\mathrm{P}=0.036)$. In addition, in terms of $\mathrm{BMI}$, no statistically significant differences were observed between the groups $(\mathrm{P}=0.841)$. In terms of the pre-menopausal state, a statistically significant difference was found between the endometrial cancer group and other groups $(\mathrm{P}=0.001)$. In total, 
Table I. Characteristics of the study subjects.

\begin{tabular}{|c|c|c|c|c|c|}
\hline $\begin{array}{l}\text { Baseline characteristics } \\
\text { of the } 4 \text { groups (no.) }\end{array}$ & $\begin{array}{l}\text { Endometrial } \\
\text { polyp }(n=7)\end{array}$ & $\begin{array}{l}\text { Submucosal } \\
\text { fibroids }(n=8)\end{array}$ & $\begin{array}{l}\text { Endometrial } \\
\text { cancer }(n=5)\end{array}$ & $\begin{array}{l}\text { Normal } \\
\text { endometrium }(n=2)\end{array}$ & P-value \\
\hline FIGO stage & & & $\begin{array}{c}\text { EC, G1 }(n=3), \\
\text { EC, G2 }(n=1), \\
\text { EC, G3 }(n=1) \\
\text { IA }(n=3) \\
\text { IB }(n=1) \\
\text { IIIA }(n=1)\end{array}$ & & \\
\hline $\begin{array}{l}\text { Age at diagnosis } \\
\text { Means } \pm \text { SD } \\
\text { Median (range) }\end{array}$ & $\begin{array}{c}38.0 \pm 3.27 \\
37.0(35-44)\end{array}$ & $\begin{array}{c}42.3 \pm 5.42 \\
42.5(35-52)\end{array}$ & $\begin{array}{c}53.0 \pm 15.2 \\
54.0(28-67)\end{array}$ & $\begin{array}{c}37.0 \pm 11.3 \\
37.0(29-45)\end{array}$ & 0.036 \\
\hline $\begin{array}{l}\text { Nulliparous } \\
\text { Yes } \\
\text { No }\end{array}$ & $\begin{array}{l}2 \\
5\end{array}$ & $\begin{array}{l}2 \\
6\end{array}$ & $\begin{array}{l}2 \\
3\end{array}$ & $\begin{array}{l}1 \\
1\end{array}$ & 1.000 \\
\hline $\begin{array}{l}\text { Pre-menopause } \\
\text { Yes } \\
\text { No (post-menopause) }\end{array}$ & $\begin{array}{l}7 \\
0\end{array}$ & $\begin{array}{l}8 \\
0\end{array}$ & $\begin{array}{l}1 \\
4\end{array}$ & $\begin{array}{l}2 \\
0\end{array}$ & 0.001 \\
\hline $\begin{array}{l}\text { BMI } \\
\text { Mean } \pm \text { SD } \\
\text { Median (range) }\end{array}$ & $\begin{array}{c}22.7 \pm 3.00 \\
23.0(18.6-26.5)\end{array}$ & $\begin{array}{c}21.5 \pm 2.80 \\
21.4(17.8-25.4)\end{array}$ & $\begin{array}{c}22.8 \pm 4.43 \\
21.8(17.9-28.6)\end{array}$ & $\begin{array}{c}23.8 \pm 7.00 \\
23.8(18.8-28.7)\end{array}$ & 0.841 \\
\hline
\end{tabular}

Values for age and BMI are presented as the means \pm standard deviation. EC, endometrioid carcinoma.

4 of 5 patients with endometrial cancer were in the post-menopausal state. Four cases had a history of gynecological surgery (ovarian cystectomy, $n=3$; and myomectomy, $n=1$ ). Two had suffered essential hypertension. One had a history of diabetes (data not shown).

Expert reviews of Hysmartscopic images. From the beginning of the procedure, the average time required to capture a video was approximately $1 \mathrm{~min}$. The system benefits from a large field of view up to 90 degrees for a single image at a distance of $3-10 \mathrm{~mm}$ from the lesions. This field aperture is comparable to the measured resolution associated with the conventional hysteroscopes (Karl Storz SE \& Co. KG). Images are captured by an 8 megapixel camera with autofocus. The data acquisition procedure is similar to that of conventional hysteroscopy. Hysmartscopy has the advantages of flexibility for allowing examiners to manipulate the scope in a hand-held manner. Two examiners reported feeling somewhat too comfortable using a smartphone (4.0/5.0 on a 5-point Likert scale). The images were acceptable for diagnostic procedures.

Diagnostic accuracy of Hysmartscopy in the detection of intrauterine abnormalities. The diagnostically important frames extracted from the Hysmartscopy iPhone videos are presented in Fig. 2. The pathological diagnosis of intrauterine lesions in 22 women with abnormal uterine cavities was as follows: Endometrial polyp ( 7 cases, $31.8 \%$ ), submucosal fibroids ( 8 cases, $36.4 \%$ ), endometrial cancer (5 cases, $22.7 \%$ ) and normal endometrium (2 cases, 9.1\%) (Table I). This study evaluated whether Hysmartscopy provides an
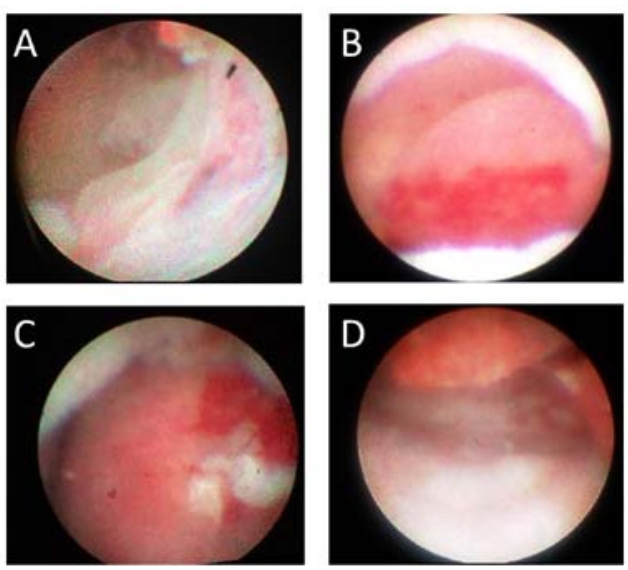

Figure 2. Visualization of abnormal pathologies in the uterine cavity. Representative intrauterine video images taken with this system are shown. (A) Normal endometrium in a 29-year-old woman. (B) Endometrial polyp (18 $\mathrm{mm}$ in diameter) in a 37-year-old woman. (C) Submucosal fibroids (29 $\mathrm{mm}$ in diameter) in a 41-year-old woman. (D) Endometrial cancer IA, $\mathrm{G} 1$, in a 54-year-old woman.

accurate pathological diagnosis. Ultimately, the results of Hysmartscopy and pathological reports of endometrial specimens were compared, and the diagnostic accuracy of Hysmartscopy for normal endometrium, endometrial polyps, submucosal fibroids and endometrial cancer was evaluated. The diagnostic sensitivity of Hysmartscopy was found to be $100 \%(2 / 2)$ for the normal endometrium, $71.4 \%(5 / 7)$ for endometrial polyps, $62.5 \%$ (5/8) for submucosal fibroids and 
Table II. The diagnostic accuracy of Hysmartscopy in the detection of intrauterine abnormalities.

\begin{tabular}{|c|c|c|c|c|c|c|}
\hline \multirow[b]{2}{*}{$\begin{array}{l}\text { Pathological } \\
\text { reports }\end{array}$} & & \multicolumn{4}{|c|}{ Hysmartscopy diagnosis } & \multirow[b]{2}{*}{$\begin{array}{c}\text { Diagnostic } \\
\text { accuracy (\%) }\end{array}$} \\
\hline & & $\begin{array}{c}\text { Normal } \\
\text { endometrium }\end{array}$ & $\begin{array}{l}\text { Endometrial } \\
\text { polyps }\end{array}$ & $\begin{array}{l}\text { Submucosal } \\
\text { fibroids }\end{array}$ & EC & \\
\hline Normal endometrium & 2 & 2 & 0 & 0 & 0 & 100 \\
\hline Endometrial polyps & 7 & 1 & 5 & 1 & 0 & 71.4 \\
\hline Submucosal fibroids & 8 & 3 & 0 & 5 & 0 & 62.5 \\
\hline \multirow[t]{2}{*}{ Endometrial cancer } & 5 & 0 & 0 & 0 & 5 & 100 \\
\hline & & & & & Total & 77.3 \\
\hline
\end{tabular}

Diagnostic agreement was evaluated between histology vs. Hysmartscopy. EC, endometrioid carcinoma.

$100 \%(5 / 5)$ for endometrial cancer (Table II). The diagnostic accuracy of Hysmartscopy in the detection of all intrauterine abnormalities was $77.3 \%$.

Adverse effects. In the first cohort, there were no complications during and after the diagnostic procedures. In the second cohort, the Hysmartscopy system was successfully performed in all 5 subjects without anesthesia. All 5 patients tolerated the procedure without analgesics or anesthesia in an office setting. None of the cases had any complications during and after diagnostic procedures.

\section{Discussion}

To the best of our knowledge, this is the first proof of concept pilot study of a novel smartphone-based ultrathin flexible fiberscope (currently known as Hysmartscopy). For this, a flexible fiberscope $(0.8 \mathrm{~mm}$ in diameter $)$, its connector, iPhone $6 \mathrm{~S}^{\mathrm{TM}}$ and portable LED light source are needed. The Hysmartscopy system used in this study is the prototype for the device. The feasibility, effectiveness, accuracy and performance of this system for diagnosing intrauterine abnormalities were demonstrated in ex vivo and clinical studies in the inpatient hospital setting and outpatient office setting.

The aim of this study was to assess the feasibility and safety of the Hysmartscopy system to diagnose intrauterine abnormalities. In the first cohort, Hysmartscopy was performed in a conventional operating room under general anesthesia in 17 patients with an abnormal uterine cavity. In the second cohort, Hysmartscopy was further validated in 5 additional subjects without anesthesia and cervical dilatation in an office setting. In the proof of concept pilot study, this system offers the advantage of direct visualization of the uterine cavity without anesthesia in the outpatient clinic. Endometrial polyps, submucosal fibroids and endometrial cancer were correctly predicted in 5/7 (71.4\%), 5/8 (62.5\%) and 5/5 (100\%) women, respectively. This study reports a high diagnostic sensitivity (77.3\%) and positive agreement between the Hysmartscopy results and the histological findings. The diagnostic accuracy of submucosal fibroids may depend on the degree of the fibroid protruding towards the endometrial cavity, which causes false endometrial thickening. The diagnostic sensitivity for endometriotic cancer $(\mathrm{n}=5)$ was $100 \%$. Bourdel et al reported that the sensitivity and specificity of endometrial cancer were 86.6 and $87.3 \%$, respectively, for experts with regarding to evaluating the diagnostic accuracy of traditional hysteroscopy (8). Hysmartscopy and conventional hysteroscopy may be equally effective in diagnosing intrauterine abnormalities.

In general, complications of surgical hysteroscopic procedures may include simply, difficulty in entering the internal os, bleeding, cervical laceration, uterine perforation, infection, fluid overload, absorption of irrigation solutions, dilutional hyponatremia, incomplete resection, intrauterine adhesions and, rarely, gas or air embolism $(2,9,10)$. The reported incidence of complications is 1 to $3 \%$, and the majority of these occur during surgical procedures (11). The severe complication rate of office hysteroscopy is rare, $0.05 \%$ (12). Hysmartscopy is diagnostic hysteroscopy and is regarded as a safe and well-tolerated procedure with rare complications. Due to the small diameter of the instrument, flexibility and maneuverability, Hysmartscopy can be used without anesthesia and cervical dilatation, and produces minimal to no trauma to the cervical canal and thus markedly diminishes any accidental bleeding.

This study also investigated the satisfaction of gynecologists and the convenience of the smart device-based Hysmartscopy. On average, the quality of the images and videos taken by expert gynecologic endoscopists may be largely sufficient to detect the emergent findings (4.0/5.0 on a 5-point Likert scale). Image acquisition and spatial resolution would be equivalent to an existing surgical camera system; the expert reviewers found the overall image quality to be acceptable for diagnostic purposes. Smartphone-based imaging can be an effective, rapid and easy to learn procedure for medical trainees or residents to capture intrauterine images in an office setting. This system is lightweight, easy to use, has a low manufacturing cost, field-portability and accessible design, together with the connectivity of smartphones, which presents a promising platform for diagnostic procedures in a variety of clinical settings. Therefore, the Hysmartscopy system is considered to be an available option for remote imaging via a network connection by an internet web-based browser.

Furthermore, flexible hysteroscopes have been developed for both diagnostic and therapeutic procedures. These scopes provide specific advantages over rigid hysteroscopes 
in wide $120^{\circ}$ field of view; however, the insertion tube outer diameter and distal end outer diameter of the insertion portion are 4.5 and $4.9 \mathrm{~mm}$, respectively (https://medical. olympusamerica.com/products). An insertion tube diameter of the slimmest hysteroscope (HYF-XP from Olympus Co., Ltd.) for office diagnostic hysteroscopy is $3.1 \mathrm{~mm}$ (https://www.olympus-europa.com/medical/rmt/media/Content/ Content-MSD/Documents/Brochures/hyf-xp__product-brochure EN_20000101.pdf). Continued technological advances will prompt the development of the less complex and slimmer device, and allow its adaptation to a minimally invasive hysteroscopic approach.

Guan et al (13) reported the advantages of robotic techniques in hysteroscopic-assisted procedure to educate surgeons. These advances could offer great value for surgeons by providing visual, navigational, and mechanical computerized assistance. The use of robotic-assisted hysteroscopy required for the intrauterine operation is growing rapidly, driven by advances in technology, which would make the difficult surgical techniques easier, safer, more accessible and more efficient. Although robotic surgery has great potential and may play an important role in surgical settings, this procedure is still a pilot instrument that needs to be improved through future studies.

The incidence of uterine cavity abnormalities detected by Hysmartscopy in this study was only $77.3 \%$. As shown in Table II, 3 cases of women with submucosal fibroids had completely normal intracavitary findings, as they had submucosal fibroids with low fibroid protrusion ratio into the uterine cavity. Not only Hysmartscopy, but also conventional hysteroscopy could not confidently diagnose submucosal fibroids with small intracavitary component, such as type II fibroids (14).

In addition, this study was limited by the small sample size $(n=22), 2$ expert gynecological endoscopists and by the use of a subjective tool of a 5-step Likert scale. However, this is a pilot proof of concept feasibility study and the 2 evaluators were blinded to the pathological results, apart from the presence of intrauterine abnormalities, which could sometimes compensate for the small size. Another limitation is the lack of comparison with an existing conventional hysteroscopy $(15,16)$. Despite these limitations, it can be considered that this new system is an accessible device, comfortable to be used by examiners and patients, and may be useful in the outpatient clinic for the direct visualization of the uterine cavity. It may complement the less accurate methods, such as blind endometrial cytology and curettage. These preliminary results warrant further investigation to evaluate whether Hysmartscopy affects therapeutic decision making and is considered the gold standard for diagnosis of any intrauterine lesion.

In conclusion, this system may be a promising and valuable alternative to the conventional hysteroscopy due to its simplicity, accuracy, well-tolerability, portability, efficient data collection and enhancing communication among physicians.

\section{Acknowledgements}

The authors would like to thank Mrs. Sachiko Minakawa (CEO, OK Fiber Technology Co., Ltd., Kyoto, Japan.) and Dr Takeshi Seki (Lecturer, Graduate School of Engineering Science, Akita University, Akita, Japan.) for providing assistance with the design, processing and assembly work accompanying the development of prototypes for the Hysmartscopy system.

\section{Funding}

No funding was received.

\section{Availability of data and materials}

All data generated or analyzed during this study are included in this published article or are available from the corresponding author on reasonable request.

\section{Authors' contributions}

KO designed a novel smartphone-based ultrathin flexible hysterofiberscopy system. KI and HS collected clinical data. HK contributed to the conception, design and interpretation of the data of this study. HK wrote the first draft. The final version of the manuscript has been read and approved by all authors.

\section{Ethics approval and consent to participate}

The study protocol was in agreement with the Helsinki Declaration for Ethical Medical Research (no. 874). Research was performed after obtaining approval from the Nara Medical University Institutional Review Board (IRB). A written informed consent was signed by each participant following a detailed explanation of the objectives and protocol of the study.

\section{Patient consent for publication}

Not applicable.

\section{Competing interests}

The authors declare that they have no competing interests.

\section{References}

1. Dueholm M, Lundorf E, Hansen ES, Ledertoug S and Olesen F: Evaluation of the uterine cavity with magnetic resonance imaging, transvaginal sonography, hysterosonographic examination, and diagnostic hysteroscopy. Fertil Steril 76: 350-357, 2001.

2. Jansen FW, Vredevoogd CB, van Ulzen K, Hermans J, Trimbos JB and Trimbos-Kemper TC: Complications of hysteroscopy: A prospective, multicenter study. Obstet Gynecol 96: 266-270, 2000

3. Gambadauro P, Martínez-Maestre MA and Torrejón R: When is see-and-treat hysteroscopic polypectomy successful? Eur J Obstet Gynecol Reprod Biol 178: 70-73, 2014.

4. Hauge K, Ekerhovd E and Granberg S: Abnormal uterine bleeding refractory to medical therapy assessed by saline infusion sonohysterography. Acta Obstet Gynecol Scand 89: 367-372, 2010.

5. Gambadauro P and Magos A: Pain control in hysteroscopy. Finesse, not local anaesthesia. BMJ 340: c2097, 2010.

6. Campo R, Santangelo F, Gordts S, Di Cesare C, Van Kerrebroeck H, De Angelis MC and Di Spiezio Sardo A: Outpatient hysteroscopy. Facts Views Vis Obgyn 10: 115-122, 2018.

7. Shigetomi H, Oka K, Seki T and Kobayashi H: Design and preclinical validation of the composite-type optical fiberscope for minimally invasive procedures of intrauterine disease. J Minim Invasive Gynecol 22: 985-991, 2015. 
8. Bourdel N, Modaffari P, Tognazza E, Pertile R, Chauvet P, Botchorishivili R, Savary D, Pouly JL, Rabischong B and Canis M: Does experience in hysteroscopy improve accuracy and inter-observer agreement in the management of abnormal uterine bleeding? Surg Endosc 30: 5558-5564, 2016.

9. van Dongen $\mathrm{H}$, de Kroon CD, Jacobi CE, Trimbos JB and Jansen FW: Diagnostic hysteroscopy in abnormal uterine bleeding: A systematic review and meta-analysis. BJOG 114 664-675, 2007

10. Aas-Eng MK, Langebrekke A and Hudelist G: Complications in operative hysteroscopy-is prevention possible? Acta Obstet Gynecol Scand 96: 1399-1403, 2017.

11. Sahu L, Tempe A and Gupta S: Hysteroscopic evaluation in infertile patients: A prospective study. Int J Reprod Contracept Obstet Gynecol 1: 37-41, 2012.

12. Capmas P, Pourcelot AG, Giral E, Fedida D and Fernandez H: Office hysteroscopy: A report of 2402 cases. J Gynecol Obstet Biol Reprod (Paris) 45: 445-450, 2016.
13. Guan Z, Liu J, Bardawil E and Guan X: Surgical management of cesarean scar defect: The hysteroscopic-assisted robotic single-site technique. J Minim Invasive Gynecol: Jun 17, 2019 (Epub ahead of print).

14. Munro MG, Critchley HO and Fraser IS; FIGO Menstrual Disorders Working Group: The FIGO classification of causes of abnormal uterine bleeding in the reproductive years. Fertil Steril 95: 2204-8, 2208.e1-3, 2011.

15. Marsh F and Duffy S: The technique and overview of flexible hysteroscopy. Obstet Gynecol Clin North Am 31: 655-668, 2004.

16. Cicinelli E: Hysteroscopy without anesthesia: Review of recent literature. J Minim Invasive Gynecol 17: 703-708, 2010.

(7) (9) This work is licensed under a Creative Commons

EY No No Attribution-NonCommercial-NoDerivatives 4.0 International (CC BY-NC-ND 4.0) License. 